G. M. S. Nys

M. J. E. van Zandvoort

A. Algra

L. J. Kappelle

E. H. F. de Haan

\title{
Cognitive and functional outcome after intravenous recombinant tissue plasminogen activator treatment in patients with a first symptomatic brain infarct
}

\author{
A. Algra, $\mathrm{MD}, \mathrm{PhD}$, FAHA \\ Julius Centre for Health Sciences \\ and Primary Care \\ University Medical Centre \\ Heidelberglaan 100 \\ 3584CX Utrecht, The Netherlands
}

Abstract Objective To examine whether intravenous recombinant tissue plasminogen activator (rt$\mathrm{PA}$ ) treatment given in the acute phase of ischaemic stroke has a favourable effect on cognitive and functional outcome at six months post-stroke. Methods The present study included 92 patients with a first-ever symptomatic infarct, of whom $25(27 \%)$ were subjected to rt-PA treatment in the first three hours post-stroke. Multivariate logistic regression analyses adjusted for stroke severity, education, age, and sex were performed to examine whether rt-PA treat- ment influenced cognitive outcome (assessed with a neuropsychological examination covering 7 cognitive domains), basic ADL independence (modified Barthel Index $\geq 19$ ), and instrumental ADL independence (Frenchay Activities Index $\geq 15$ ) after six months. Results The adjusted odds ratio for intact cognition was $1.0(95 \% \mathrm{CI}$ 0.2 to 4.3 ), that for basic ADL outcome 13.5 (95\% CI 1.4 to 129.4 ) and for instrumental ADL 7.1 (95\% CI 1.2 to 42.2 ). Conclusion Our findings suggest that rt-PA treatment is associated with a favourable basic and instrumental ADL outcome, but not with a beneficial cognitive outcome after 6 months.

Key words thrombolysis - acute stroke $\cdot$ cognition $\cdot$ functional outcome

\section{Introduction}

Patients treated with intravenous recombinant tissue plasminogen activator (rt-PA) within the first three hours after stroke have a favourable clinical outcome at three months post-stroke as compared with patients treated with placebo $[1,2]$. Similar treatment effects have been found after six months and one year poststroke [3]. The benefit of rt-PA is thought to be due to the early recanalisation of occluded arteries, resulting in increased cerebral reperfusion [4] and a subsequent reduction of lesion volume [5]. The sooner thrombolytic treatment is administered after stroke, the greater the efficacy of the treatment $[6,7]$. Although the majority of studies report a beneficial effect of rt-PA, there are also some reports of adverse effects, such as an increased mortality and intracerebral haemorrhage in the first week post-stroke [1], and neurotoxic effects of rt-PA via potentiation of excitotoxicity when recanalisation after treatment is unsuccessful [8].

Cognitive impairment is known to be strongly associated with an adverse functional outcome [9] and with post-stroke dementia [10]. Therefore, it is useful to evaluate whether rt-PA treatment might reduce the prevalence of cognitive impairment after stroke. Moreover, 
while it has been shown that patients treated with rt-PA have a favourable outcome with respect to basic activities of daily life (bADL) [1-3], it is unknown whether this also applies to more complex activities that are necessary for independence at home (instrumental ADL: iADL).

The aim of this study was to examine if intravenous rt-PA treatment in the first three hours post-stroke has a beneficial effect on cognitive and functional outcome after six months, independent of demographic factors and stroke severity at baseline.

\section{Methods}

\section{Patients}

Patients with a first-ever symptomatic ischaemic stroke admitted to dedicated stroke units of three hospitals in The Netherlands (University Medical Centre Utrecht, St. Elisabeth Hospital Tilburg, and Tweesteden Hospital Tilburg) between January 2002 and July 2004 were eligible for inclusion in the present study. The diagnosis of stroke was based on the presence of both an acute focal deficit and an associated lesion on CT or MRI. Patients with a normal scan underwent a second scan within the first week post-stroke. We excluded patients with a neurological or psychiatric history, with a history of preexistent cognitive deterioration (as defined by a score of 3.6 or higher on the short Informant Questionnaire on Cognitive Decline in the Elderly - IQCODE Dutch Version) [11], and patients who were admitted to the hospital $>24$ hours following the first symptoms. Patients older than 85 years were also excluded to prevent disproportional aging effects on cognitive performance. We applied no exclusion criteria with regard to the severity of post-stroke cognitive impairment, and both patients with aphasia and unilateral neglect were included. In the University Medical Centre Utrecht, intravenous administration of rt-PA was supplied to all patients arriving at the stroke unit within three hours after stroke, if they did not have exclusion criteria for thrombolysis as recommended in the NINDS study [1]. The other two participating centres (St. Elisabeth \& Tweesteden Hospital) had not yet provided rt-PA at the time of data collection. Apart from the rt-PA intervention, stroke treatment was similar in the three stroke units and standardised according to the same protocol. Moreover, the available rehabilitation programs were comparable across the three centres. On the whole, 25 patients received thrombolytic treatment and 67 patients did not. A control group was assembled as a reference sample for the neuropsychological examination, consisting of 75 subjects living in the community. The controls were either spouses or family of patients, or volunteers who came to our attention through advertising in newspapers or by word of mouth. Control subjects were carefully matched to the stroke patients with respect to age, education, and gender [9].

The ethics committee of the three hospitals approved the study protocol. Informed consent was obtained from all participating subjects before inclusion in the study.

\section{Baseline data}

Stroke severity at the time of admission on the stroke unit was obtained from the medical files by means of the National Institutes of Health Stroke Scale (NIHSS) [12]. In addition, demographic factors were recorded, i. e. age, sex, and level of education (scored with 7 categories ranging from 1: did not finish primary school to 7: university degree, and dichotomised at the median) [9].

An extensive neuropsychological examination was administered after a minimum of 6 and a maximum of 10 months post-stroke, and was performed by the same investigators at all centres. The examina- tion comprised 7 cognitive domains [9, 13]: (1) abstract reasoning [Raven Advanced Progressive Matrices (short form), Similarities (WAIS-III)] (2) verbal memory [Rey Auditory Verbal Learning Test, Wechsler Memory Scale - story recall A, Digit span (WAIS-III)] (3) executive functioning [Brixton Spatial Anticipation Test, the Visual Elevator (Test of Everyday Attention), letter fluency, the Stroop Colour Word test, Zoo test (BADS)] (4) visual perception and construction [Judgment of Line Orientation (short form), the Test of Facial Recognition (short form), WAIS-III block patterns] (5) visual memory [Wechsler Memory Scale-Visual Reproduction, Location Learning Task, Corsi Block span] (6) language [Token Test (short form), Boston Naming test (short form), Chapman reading task], and (7) unilateral neglect [Star Cancellation]. The procedure of administering multiple neuropsychological tasks within one cognitive domain allowed us to transform raw test scores of patients on individual tasks into compound $\mathrm{z}$-scores based on the means and standard deviations of the control group. Subsequently, we averaged $z$-scores of tasks belonging to the same cognitive domain [9]. Cut-off scores for cognitive impairment within each domain were determined by a performance that differed from the control mean at the 0.05 level of significance ( $\mathrm{z}-$ score $<-1.65)$ [13].

\section{Definition of outcome measures}

Cognitive outcome as assessed with the above-mentioned neuropsychological examination was classified as (i) 'cognitively intact' [defined as no impairment on any of the cognitive domains] vs. 'cognitively impaired' [defined as an impairment in at least one cognitive domain] and (ii) severity of cognitive impairment [cognitive compound score which is the unweighted average of the seven cognitive domain scores].

Functional outcome was assessed with two separate ADL measures, that is basic and instrumental ADL measures. Basic ADL instruments assess straightforward activities such as personal hygiene and dressing, whereas instrumental ADL instruments assess more complex activities, such as grocery shopping, household management, and social activities. In this study, basic ADL was determined with the modified Barthel Index (mBI) [14]. An mBI value $\geq 19$ was used as an indication of intact basic ADL function [9]. The Frenchay Activities Index (FAI) [15] was used to assess instrumental ADL. In total, the scale comprises 15 individual activities summed to give an overall score ranging from 0 (inactive) to 45 (very active). Intact instrumental ADL function was defined as FAI $\geq 15$ [9].

\section{Statistical analyses}

The primary aim of the analyses was to compare the prevalence of intact cognition and basic and instrumental ADL independence after six months between the patients treated with rt-PA and those not treated. To this end we calculated crude and adjusted odds ratios (OR) with corresponding $95 \%$ confidence intervals $(95 \% \mathrm{CI})$ by means of logistic regression. We adjusted for age, sex, level of education, and NIHSS at baseline. In addition we compared the mean cognitive compound score between the patients treated with rt-PA and those not treated by means of linear regression adjusting for the same four factors.

\section{Results}

Patient characteristics are shown in Table 1. In general, patients who received rt-PA treatment demonstrated a more severe stroke.

An intact cognitive outcome after six months was 
Table 1 Patient characteristics $(\mathrm{N}=92)$

\begin{tabular}{lll}
\hline & no rt-PA (N =67) & rt-PA (N=25) \\
\hline $\begin{array}{l}\text { Interval stroke onset to outcome } \\
\text { examination, months }\end{array}$ & $7.7 \pm 1.4$ & $8.0 \pm 2.2$ \\
Age, years & $61.7 \pm 12.7$ & $59.9 \pm 13.9$ \\
High education*, $\%$ & $43 \%$ & $64 \%$ \\
Female sex, \% & $43 \%$ & $24 \%$ \\
NIHSS, median (range) & $5(2-17)$ & $11(2-18)$ \\
\hline
\end{tabular}

Values are means \pm standard deviations unless otherwise indicated. ${ }^{*}$ High education $=5$ (high school education) to 7 (university degree)

present in $52 \%$ of patients treated with intravenous rtPA vs. $66 \%$ of patients who were not treated with rt-PA (adjusted OR 1.0; $95 \%$ CI 0.2 to 4.3) (Table 2). Basic ADL independence was found in respectively $88 \%$ and $82 \%$ of patients (adjusted OR 13.5; 95\% CI 1.4 to 129.4), and instrumental ADL independence was observed in respectively $83 \%$ and $80 \%$ (adjusted OR 7.1; $95 \%$ CI 1.2 to 42.2). Treatment with rt-PA exerted no positive effect on the severity of cognitive impairment (adjusted difference $0.01 ; 95 \% \mathrm{CI}-0.4$ to 0.6 ).

Next, in order to further examine the potential confounding influence of stroke severity in our study, we compared the 25 patients with the highest NIHSS scores (6-17) who were not treated with the 25 patients who were treated (2-18) (total group $\mathrm{N}=50$ ). We performed the same regression analyses as before, yielding the same absence of treatment effect with respect to cognition (adjusted OR 0.9), but instead an even more profound treatment effect with respect to basic ADL (adjusted OR 47.1) and instrumental ADL (adjusted OR 12.7).

\section{Discussion}

This is the first report on cognitive and instrumental ADL outcome after treatment with intravenous rt-PA administered in the first three hours post-stroke. Our findings suggest that rt-PA treatment is associated with a favourable basic and instrumental ADL outcome, but not with a beneficial cognitive outcome at 6 months. This sample included only patients who survived a firstever symptomatic stroke. Therefore, these findings cannot be directly generalised to the stroke population as a whole. Furthermore, it was not possible to randomise treatment. Patients did not receive treatment either because they were admitted to one of the two participating centres in Tilburg (The Netherlands), because they were excluded by means of the NINDS criteria for thrombolytic treatment [1], or because they arrived too late at the hospital ( $>3$ hours post-stroke). As a result, patients subjected to rt-PA in our study demonstrated more severe strokes than the untreated patients, in line with findings from a previous study in which patients with more severe strokes arrived earlier in the emergency departments than those whose condition was less severe [7]. Risk adjustment statistically addresses this heterogeneity and has been shown to reduce bias in treatment effect estimates [16]. Nevertheless, there might be some residual confounding that diminishes the estimated effect of treatment in our study. Therefore, we re-ran our analyses with comparable groups with regard to stroke severity and obtained essentially the same results, suggesting that a potential bias in stroke severity cannot fully explain our results. Moreover, functional outcome six months after stroke was better in those treated despite greater stroke severity, with respect to both basic and instrumental ADL. This is an important finding suggesting that rt-PA treatment not only affects straightforward activities such as personal hygiene and dressing, but also more complex activities such as grocery shopping and household management. Independence in these activities enables the stroke patient to be discharged home without being too dependent on others. With respect to basic functional outcome, our findings replicate and extend a previous study on the benefit of rt-PA at 6 and 12 months post-stroke [3].

In contrast to our findings concerning functional
Table 2 Crude and adjusted relationships of rt-PA treatment with cognition and ADL

\begin{tabular}{|c|c|c|c|c|}
\hline \multirow[b]{2}{*}{ Outcome } & \multicolumn{2}{|c|}{ Crude relationship } & \multicolumn{2}{|c|}{ Adjusted $^{*}$ relationship } \\
\hline & OR/Beta** & $95 \% \mathrm{Cl}$ & OR/Beta** & $95 \% \mathrm{Cl}$ \\
\hline Intact cognition & 0.6 & 0.2 to 1.4 & 1.0 & 0.2 to 4.3 \\
\hline Cognitive compound score & -0.2 & -0.6 to 0.3 & 0.01 & -0.4 to 0.6 \\
\hline bADL independence & 1.6 & 0.4 to 6.2 & 13.5 & 1.4 to 129.4 \\
\hline iADL independence & 1.3 & 0.4 to 4.4 & 7.1 & 1.2 to 42.2 \\
\hline
\end{tabular}

* Adjusted for age, sex, level of education, and NIHSS

** All data are odds ratios derived from logistic regression, except for the cognitive compound score which are betas derived from linear regression. An odds ratio $<1$ denotes fewer patients with intact cognition or ADL independence in the patients treated with rt-PA. Beta indicates the difference in the mean cognitive compound score between the patients treated and not treated with rtPA. Beta $<0$ denotes worse cognitive compound scores among the rt-PA treated patients 
outcome, we could not demonstrate an effect of rt-PA treatment on the prevalence or the severity of cognitive impairment six months after stroke. Three alternative explanations may be given for this finding. First, it is possible that treatment with rt-PA does not affect cognitive outcome at six months. This explanation would suggest that reducing the volume of the lesion is not sufficient to ameliorate cognitive functioning in the long term after stroke. Other factors, such as the location of the lesion [17], the volume of hypoperfusion [18], or neuronal metabolic changes [19] have indeed been shown to be equally or more important for cognitive recovery after stroke. Alternatively, it is possible that rt-PA reduces lesion volume resulting in an improvement of focal cognitive functioning, but that, at the same time, rt-PA is neurotoxic [8] resulting in a concurrent global cognitive decline in some cases. A second explanation for our finding regarding cognitive outcome may be that rt-PA treatment has a short-term influence on cognitive outcome, but that this effect is not sustained at six months. For instance, the rt-PA group may show a better cognitive outcome after 1 month than patients who are not treated with rt-PA, but this difference might disappear in the long term due to a gradual recovery in untreated patients resulting in the same cognitive outcome after six months. The third explanation concerns the design of the study. Perhaps the statistical power was insufficient to demonstrate a positive effect of rt-PA on cognitive outcome, or the effect is masked by the abovementioned residual confounding of baseline differences between the two patient groups. The latter explanation seems less likely given our findings with respect to basic and instrumental functional outcome.
A possible limitation of this study is that the NIHSS stroke scale demonstrates a lateralization bias, i. e. prior research has shown that it is a poor measure of stroke severity in patients with cognitive dysfunction due to right hemispheric stroke [20]. Therefore stroke severity may have been underestimated in patients with a right hemispheric stroke in our study. Nevertheless, it is unlikely that this lateralisation bias would exert a differential influence on the different outcome measures. In any case, future studies with randomised and larger stroke samples are needed to examine the short- and long-term effects of thrombolytic treatment on cognitive functioning, and it would probably be more suitable to administer a stroke severity scale of which the score relies equally on functions of the left and the right hemisphere. Moreover, as the treatment is only safe and effective within the first hours post-stroke [6,7], a revolution in neurological services is needed with an emphasis on immediate care. In addition, public education is essential in order to improve knowledge about symptoms of acute stroke.

In sum, our findings suggest that rt-PA treatment influences basic and functional outcome, but not cognitive outcome after six months. The findings should be confirmed in a population more representative of the patients who are typically enrolled in acute stroke trials. In the meantime, the search for appropriate neuropsychological interventions aimed at reducing cognitive impairment after stroke remains crucial.

Acknowledgements We thank dr. P.L.M. de Kort and dr. B.P.W. Jansen for the enrolment of patients, and Claire de Monte for her assistance with the data collection. This study was funded by the Netherlands Heart Foundation (nr. 2000.023).

\section{References}

1. The National Institute of Neurological Disorders and Stroke rt-PA Stroke Study Group (1995) Tissue plasminogen activator for acute ischemic stroke. N Engl J Med 333:1581-1587

2. Hacke W, Kaste M, Fieschi C, Toni D, Lesaffre E, von Kummer R, et al. for the ECASS Study Group (1995) Intravenous thrombolysis with recombinant tissue plasminogen activator for acute hemispheric stroke. JAMA 274: $1017-1025$

3. Kwiatkowski TG, Libman RB, Frankel M, Tilley BC, Morgenstern LB, Lu M, et al. (1999) Effects of tissue plasminogen activator for acute ischemic stroke at one year. N Engl J Med 340: 1781-1787

4. Grotta JC, Alexandrov AV (1998) tPAassociated reperfusion after acute stroke demonstrated by SPECT. Stroke 29:429-432
5. The National Institute of Neurological Disorders and Stroke rt-PA Stroke Study Group (2000) Effect of intravenous recombinant tissue plasminogen activator on ischemic stroke lesion size measured by computed tomography. Stroke 31:2912-2919

6. Marler JR, Tilley BC, Lu M, Brott TG, Lyden PC, Grotta JC, Broderick JP, Levine SR, Frankel MP, Horowitz SH, Haley EC Jr, Lewandowski CA, Kwiatkowski TP (2000) Early stroke treatment associated with better outcome: the NINDS rt-PA stroke study. Neurology 55:1649-1655
7. Hacke W, Donnan G, Fieschi C, Kaste M, von Kummer R, Broderick JP, Brott T, Frankel M, Grotta JC, Haley EC Jr, Kwiatkowski T, Levine SR, Lewandowski C, Lu M, Lyden P, Marler JR, Patel S, Tilley BC, Albers G, Bluhmki E, Wilhelm M, Hamilton S; ATLANTIS Trials Investigators; ECASS Trials Investigators; NINDS rt-PA Study Group Investigators (2004) Association of outcome with early stroke treatment: pooled analysis of ATLANTIS, ECASS, and NINDS rt-PA stroke trials. Lancet 363:768-774

8. Busch E, Beaulieu C, de Crespigny A, Kreischer S, Diener HC, Moseley ME (2002) Combined X-ray angiography and diffusion-perfusion MRI for studying stroke evolution after rt-PA treatment in rats. Brain Res 953: 112-118 
9. Nys GMS, van Zandvoort MJE, de Kort PLM, van der Worp HB, Jansen BPW, Algra A, de Haan EHF, Kappelle LJ (2005) The prognostic value of domain-specific cognitive abilities in acute first-ever stroke. Neurology 64: 821-827

10. Lin JH, Lin RT, Tai CT, Hsieh CL, Hsiao SF, Liu CK (2003) Prediction of poststroke dementia. Neurology 61: 343-348

11. de Jonghe JF, Schmand B, Ooms ME, Ribbe MW (1997) Abbreviated form of the Informant Questionnaire on cognitive decline in the elderly. Tijdschr Gerontol Geriatr 28:224-229

12. Brott $\mathrm{T}$, Adams HP Jr, Olinger CP, Marler JR, Barsan WG, Biller J, Spilker J, Holleran R, Eberle R, Hertzberg V, et al. (1989). Measurements of acute cerebral infarction: a clinical examination scale. Stroke 20:864-870
13. Lezak MD, Howieson DB, Loring DW (2004) Neuropsychological Assessment, $4^{\text {th }}$ ed. New York: Oxford University Press

14. Mahoney FJ, Barthel DW (1965) Functional evaluation: Barthel Index. Md State Med J 14:61-65

15. Wade DT, Legh-Smith J, Langton Hewer R (1985) Social activities after stroke: measurement and natural history using the Frenchay Activities Index. Int Rehabil Med 7:176-181

16. Johnston KC, Connors AF Jr, Wagner DP, Haley EC Jr (2004) Risk adjustment effect on stroke clinical trials. Stroke 35:e43-e45
17. Zekry D, Duyckaerts C, Belmin J, Geoffre C, Herrmann F, Moulias R, Hauw JJ (2003) The vascular lesions in vascular and mixed dementia: the weight of functional neuroanatomy. Neurobiol Aging 24:213-219

18. Hillis AE, Barker PB, Wityk RJ, Aldrich EM, Restrepo L, Breese EL, Work M (2004) Variability in subcortical aphasia is due to variable sites of cortical hypoperfusion. Brain Lang 89:524-530

19. Van Zandvoort MJE, van der Grond J, de Haan EHF, Kappelle LJ (2005) Cognitive deficits and changes in neuronal metabolism after a lacunar infarct. J Neurol 252:183-90

20. Hillis AE, Wityk RJ, Barker PB, Ulatowski JA, Jacobs M (2003) Change in perfusion in acute nondominant hemisphere stroke may be better estimated by tests of hemispatial neglect than by the National Institutes of Health Stroke Scale. Stroke 34: 2392-2398 